\title{
Do Blood Transfusions Affect the Outcomes of Patients Who Underwent Elective Coronary Artery Bypass Grafting?
}

Lok Yuh Ing*, Cheng Kok Mun

Department of Cardiothoracic Surgery, Hospital Serdang, Selangor, Malaysia

DOI: $10.36347 /$ sjams.2020.v08i05.001

| Received: 26.04.2020 | Accepted: 03.05.2020 | Published: 06.05.2020

*Corresponding author: Lok Yuh Ing

Abstract

Original Research Article

Background: Red blood cell (RBC) transfusions are frequently essential in cardiac procedures to correct anaemia, coagulopathy, blood loss, and hemdilution from pump priming. However, RBC transfusions have deleterious effects of receptor immune system leading to infections after cardiac surgery. Nevertheless, it is difficult to show the direct association between RBC transfusions and postoperative morbidities or mortality due to the fact that most patients undergoing cardiac surgery have multiple comorbidities and are critically ill. Hence, we conducted a study to assess the outcomes of perioperative allogenic RBC transfusions that underwent elective coronary artery bypass graft surgery $(\mathrm{CABG})$ requiring cardiopulmonary bypass (CPB). Methods: We retrospectively analysed 130 patients who underwent first-time elective isolated coronary artery bypass operations requiring cardiopulmonary bypass between July to December 2017 at our institution. Patients with transfusions were compared with those who had no transfusion. Pre-, intra-, and postoperative data were collected from medical records, operating notes and blood transfusions database. Chi-square test and student's t-test were used, and p-value $<0.05$ was considered significant. Results: $75.3 \%$ of the patients received RBC transfusions. The median number of RBC units transfused per patient was 2 . Compared to non-RBC recipients, $\mathrm{RBC}$ recipients were associated with a higher incidence of acute kidney injury $(41 \%$ vs $60 \%$, $\mathrm{p}=0.05)$, wound infection ( $18 \%$ vs $39 \%, \mathrm{p}=0.038)$ and mortality $(0 \%$ vs $11 \%, \mathrm{p}=0.048)$. Nonetheless, there was no significant association between patients' underlying comorbidities and postoperative morbidities or mortality. Conclusions: Perioperative allogenic blood transfusions for CABG requiring CPB were significantly associated with increased post-operative morbidities and mortality.

Keywords: Transfusion, elective coronary artery bypass grafting, cardiopulmonary bypass, postoperative, infection, acute kidney injury, mortality.

Copyright @ 2020: This is an open-access article distributed under the terms of the Creative Commons Attribution license which permits unrestricted use, distribution, and reproduction in any medium for non-commercial use (NonCommercial, or CC-BY-NC) provided the original author and source are credited.

\section{INTRODUCTION}

Approximately $1,000,000$ patients are undergoing cardiac surgery worldwide annually. RBC transfusions are frequently essential in cardiac procedures to correct anaemia, blood loss, hemodilution from pump priming and coagulopathy, especially in CABG surgery due to perioperative use of potent antiplatelet drugs and the invasiveness of the procedure. The incidence of RBC transfusions among patients undergoing $\mathrm{CABG}$ surgery has been reported vary between $7.8 \%$ to $92.8 \%$ among institutions [1]. However, RBC transfusions have deleterious effects of receptor immune system leading to immunosuppression or transfusion-transmitted infections after cardiac surgery [2]. Nevertheless, it is difficult to show the direct association between transfusions and postoperative morbidities or mortality due to the fact that most patients undergoing cardiac surgery are critically ill and have multiple comorbidities e.g. anaemia, hypertension, diabetes, and chronic kidney disease. Hence, we conducted a study to assess the outcomes of perioperative allogenic RBC transfusions that underwent elective $\mathrm{CABG}$ requiring $\mathrm{CPB}$.

\section{METHODS}

Retrospective analysis of electronic medical records, operating notes and blood transfusions database for all adult patients (>18 years old) who underwent first-time elective isolated coronary artery bypass operations requiring cardiopulmonary bypass at Department of Cardiothoracic Surgery Serdang Hospital between July to December 2017. Patients who were $\leq$ 18 years old, underwent emergency CABG (the need for intervention up to 48 hours due to imminent risk of death or hemodynamically unstable), underwent CABG without $\mathrm{CPB}$, diagnosed with active systemic infection preoperatively (through laboratory tests) or, diagnosed 
with Hepatitis B, Hepatitis C or HIV infection were excluded from this study.

Baseline characteristics were obtained from electronic medical records. These included age, gender, history of hypertension, diabetes mellitus, chronic kidney disease (KDIGO guideline), ejection fraction $<55 \%$ (echocardiography performed within 6 months prior to surgery at Serdang Hospital), anaemia (haemoglobin level $<13 \mathrm{~g} / \mathrm{dL}$ for male, haemoglobin level $<12 \mathrm{~g} / \mathrm{dL}$ for female) and preoperative haemoglobin (were measured within 1 week prior to surgery). The duration of cardiopulmonary bypass and the number of cases requiring chest reopen were obtained from operative notes. Total number of perioperative allogenic RBC units transfused for each patient between 5 days before and 10 days after CABG were obtained from blood transfusions database. RBC transfusion was initiated when haemoglobin $(\mathrm{Hb})$ level $<8 \mathrm{~g} / \mathrm{dL}$ or patients in critical clinical conditions at the physician's discretion.

Three coprimary outcomes were analysed as possible complications related to RBC transfusions which were infectious outcomes, ischemic outcomes and mortality. The infectious outcomes include sepsis (diagnosed by isolated organisms in culture associated with fever), respiratory infection (diagnosed by fever with productive cough, elevated white blood cell count, and identification of lung infection in Chest X-Ray or CT thorax), wound infection (diagnosed by the presence of pain, warmth, redness and pus at the sternal wound, presence of sternal instability with fever or through CT thorax) and transfusion-transmitted infection (diagnosed by HBsAg, Hepatitis C antibody and HIV tests repeated at 42 nd week after surgery). Ischemic outcomes include stroke (diagnosed by clinical signs of neurological deficit and CT brain) and acute kidney injury (according to KDIGO guideline). Lastly, death rate that occurred during hospitalization or within 30 days after surgery was analysed.

Quantitative variables were described as mean and standard deviation and qualitative variables as absolute number and percentages. The number of unit(s) transfused per patient is expressed as the median. Independent samples t-test was performed for continuous variables whereas the chi-square test was used to compare categorical variables. The variables with $\mathrm{P}<0.05$ were considered significant. The software SPSS (Statistical Package for the Social Sciences, version 20) were used for statistical analysis.

\section{RESULTS}

From the total of 130 patients in this study, 98 patients $(75.3 \%)$ received allogenic RBC transfusions during perioperative period. Baseline characteristics of the non-transfused and transfused groups were compared in Table 1. Diabetes mellitus and cardiopulmonary bypass time were associated with RBC transfusion. Other variables were not associated with exposure to RBC transfusion. The median number of RBC units transfused per patient was 2 .

Table-1: Baseline Characteristics and Perioperative Characteristics of Patients

\begin{tabular}{|l|l|l|l|}
\hline Variables & Non-transfused (n=32) & Transfused (n=98) & P value \\
\hline Age, $\mathbf{n}(\%)$ & & & \\
\hline $\mathbf{6 5 5}$ years & $22(69)$ & $70(71)$ & 0.77 \\
\hline $\mathbf{6 5}$ years & $10(31)$ & $28(29)$ & - \\
\hline Sex, $\mathbf{n}(\%)$ & & & \\
\hline Male & $28(88)$ & $74(76)$ & 0.15 \\
\hline Female & $4(12)$ & $24(24)$ & - \\
\hline Hypertension, n(\%) & $24(75)$ & $74(76)$ & 0.95 \\
\hline Diabetes mellitus, n(\%) & $11(34)$ & $63(64)$ & 0.003 \\
\hline Chronic kidney disease, n(\%) & $7(22)$ & $31(32)$ & 0.29 \\
\hline Ejection fraction <55\%, n(\%) & $18(56)$ & $52(53)$ & 0.75 \\
\hline Preoperative haemoglobin level, g/dL & $13.0 \pm 2.12$ & $13.2 \pm 1.8$ & 0.5 \\
\hline Anaemia, n(\%) & $10(31)$ & $22(22)$ & 0.316 \\
\hline Aortic cross clamp, minutes & $102 \pm 34$ & $106 \pm 29$ & 0.566 \\
\hline Cardiopulmonary bypass time, minutes & $133 \pm 28$ & $156 \pm 41$ & 0.009 \\
\hline Chest reopen, n(\%) & $0(0)$ & $3(3)$ & 0.317 \\
\hline
\end{tabular}

Analyses of postoperative complications in relation to RBC transfusions were shown in Table 2. Patients who received blood transfusions were associated with higher incidence of acute kidney injury (41\% vs $60 \%, \mathrm{p}=0.05)$, wound infection $(18 \%$ vs $39 \%$, $\mathrm{p}=0.038)$ and mortality ( $0 \%$ vs $11 \%, \mathrm{p}=0.048)$. The causes of death were sepsis ( 7 cases), mediastinitis (2 cases), refractory bleeding (1 case) and myocardial failure (1 case). Nonetheless, complications of stroke, respiratory infection, sepsis, transfusion-transmitted infection and length of hospital stay did not differ between non- $\mathrm{RBC}$ recipients and $\mathrm{RBC}$ recipients. 
Table-2: Postoperative Complications

\begin{tabular}{|l|l|l|l|}
\hline Variables & Non-transfused $(\mathbf{n}=\mathbf{3 2})$ & Transfused $(\mathbf{n}=\mathbf{9 8})$ & P value \\
\hline Stroke, $\mathbf{n}(\%)$ & $0(0)$ & $2(2)$ & 0.42 \\
\hline Acute kidney injury, n(\%) & $13(41)$ & $59(60)$ & 0.05 \\
\hline Wound infection, $\mathbf{n}(\%)$ & $6(18)$ & $38(39)$ & 0.038 \\
\hline Respiratory infection, $\mathbf{n}(\%)$ & $0(0)$ & $2(2)$ & 0.42 \\
\hline Sepsis, $\mathbf{n}(\%)$ & $3(9)$ & $13(13)$ & 0.561 \\
\hline Transfusion transmitted infection, $\mathbf{n}(\%)$ & $0(0)$ & $0(0)$ & -- \\
\hline Hospital stay, days & $7.9 \pm 2.4$ & $9.6 \pm 6.2$ & 0.14 \\
\hline Perioperative mortality, $\mathbf{n}(\%)$ & $0(0)$ & $11(11)$ & 0.048 \\
\hline
\end{tabular}

Apart from that, analyses of comorbidities in relation to postoperative wound infection were shown in Table 3 and preoperative anaemia in relation to postoperative complications was shown in Table 4.
There was no significant association between patients' underlying comorbidities and postoperative morbidities or mortality.

Table-3: Comorbidities in relation to postoperative wound infection

\begin{tabular}{|l|l|l|l|}
\hline Variables & Wound infection $(\mathbf{n}=\mathbf{4 4})$ & No wound infection $(\mathbf{n}=\mathbf{8 6})$ & P value \\
\hline Preoperative anaemia, $\mathbf{n}(\boldsymbol{\%})$ & $10(23)$ & $22(26)$ & 0.72 \\
\hline Diabetes Mellitus, $\mathbf{n}(\boldsymbol{\%})$ & $27(61)$ & $47(55)$ & 0.47 \\
\hline Chronic kidney disease, $\mathbf{n}(\boldsymbol{\%})$ & $15(34)$ & $23(27)$ & 0.38 \\
\hline
\end{tabular}

Table-4: Preoperative anaemia in relation to postoperative complications

\begin{tabular}{|l|l|l|l|}
\hline Variables & $\begin{array}{l}\text { Preoperative anaemia } \\
(\mathbf{n}=\mathbf{3 2})\end{array}$ & Normal haemoglobin level $(\mathbf{n}=\mathbf{8 6})$ & P value \\
\hline Acute kidney injury, $\mathbf{n}(\%)$ & $17(53)$ & $55(64)$ & 0.77 \\
\hline Perioperative mortality, $\mathbf{n}(\%)$ & $3(9)$ & $8(9)$ & 0.83 \\
\hline
\end{tabular}

\section{DISCUSSION}

In our study, we found that patients who had diabetes mellitus and prolonged cardiopulmonary bypass time were more likely to receive RBC transfusions. These findings emphasize the effort to improve preoperative care and operative technique with meticulous haemostasis to avoid RBC transfusions.

Out of 130 patients underwent elective $\mathrm{CABG}$ surgery, none of the patients received RBC transfusion during preoperative period, all patients had $\mathrm{RBC}$ transfusions during either intraoperative or postoperative period. The usual RBC transfusion indications are based on transfusion triggers as well as hemodilution due to heavy fluid load in extracorporeal circulation [3]. Thus, haemoglobin alone may be an inaccurate method of replacing red cell volume loss on CPB. Slight et al. found that considering haemoglobin concentration alone may overestimate the requirement for red cell transfusion in elective cardiac surgery patients. Patients transfused as per the red cell volumebased guideline received significantly less red cells with no associated difference in clinical outcome [4].

In this study, the median number of RBC units transfused per patient was 2 . Owing to this finding, we have reduced preoperative $\mathrm{RBC}$ order for elective CABG from 4 units to 2 units since year 2018. This allowed proper blood management at blood bank in which blood products were distributed to patients who are in need and hence reduced unnecessary wastage of blood products.

Our study showed direct association of RBC transfusions with high incidence of postoperative wound infection, acute kidney injury and mortality. Meanwhile, there was no significant association between patients' underlying comorbidities and postoperative morbidities or mortality. In a study conducted by Koch et al. [5], the authors also concluded that each administered unit increases by $77 \%$ the postoperative mortality risk and $100 \%$ the risk of developing any renal complication, $76 \%$ the risk of developing postoperative infections, cardiac complications in $55 \%$ and $37 \%$ the risk of neurological complications. These associations remained strong even after the risk adjustment factors causing postoperative complications. In Magedanz study et al.[6], in which a risk score for mediastinitis was created, an association between blood transfusion and risk of postoperative mediastinitis was found (OR: 2.5; CI95\%: $1.5-4.1, \mathrm{P}=$ $0.001)$, that also agrees with a study that found a strong association between transfusion and risk of infections, which reflects the immunosuppressive effect of blood transfusion[7]. Apart from immunosuppressive effect, some studies report that blood loss and blood transfusion are known to cause severe systemic inflammatory response leading to ischemic complications by modifying the systemic inflammatory response of the patients and the direct introduction of bioactive substances into circulation [8-10]. On the 
other hand, the study conducted by Van Straten et al. [8] showed the number of units transfused may be a predictor of early mortality up to 30 days in the postoperative period, but not of late mortality. The authors concluded that in patients who received three or more units of RBC, the survival rate drops significantly when compared to non-RBC recipient. These patients have also high incidence of postoperative complications, which could explain the high early mortality in this study.

In view of blood transfusions associated with multiple postoperative complications, it is important to implement strategies for reducing $\mathrm{RBC}$ transfusions in postoperative cardiac surgery. Some authors report the importance of a careful preoperative assessment in order to reduce the risk of bleeding and blood transfusion during intraoperative and postoperative period. Apart from that, the measurement of serum iron and preoperative administration of iron and erythropoietin may reduce the need for $\mathrm{RBC}$ transfusions. Besides, autologous blood donation is used to reduce homologous blood transfusion in a wide variety of elective surgical procedures including cardiac surgeries; the patient's blood is collected prior to surgery in order to be used after the procedure [11]. Other method includes using miniEC (reduction of the prime, the tubes and vacuum drainage) and centrifugal pumps (Bio Pump) to reduce the excessive use of heparin and hence reduce the risk of bleeding $[12,13]$. Hypothermia (between $30^{\circ} \mathrm{C}$ and $32^{\circ} \mathrm{C}$ ) during the surgical procedure may also reduce bleeding during surgery and in the postoperative period.

Limitations of our study include this study was a single centre retrospective study, the sample size was relatively small and the effects of blood component transfusions other than RBC in the postoperative period were not taken into consideration.

\section{Conclusion}

Perioperative allogenic blood transfusions for CABG requiring $\mathrm{CPB}$ were significantly associated with increased post-operative wound infection, acute kidney injury and mortality.

\section{Lists of abbreviations used}

RBC - Red blood cell

$\mathrm{CPB}$ - Cardiopulmonary bypass

CABG - Coronary artery bypass grafting

CT - Computed tomography

HIV - Human immunodeficiency virus

HBsAg - Hepatitis B surface antigen

KDIGO - Kidney Disease: Improving Global Outcomes

\section{ACKNOWLEDGEMENTS}

This work was supported by the Department of Cardiothoracic Surgery, Serdang Hospital.

\section{Declaration of conflicting interests}

No conflicts of interest in preparing this article

Funding

research.

There was no external funding source for this

\section{REFERENCES}

1. Bennett-Guerrero E, Zhao Y, O'Brien SM, Ferguson TB, Peterson ED, Gammie JS, Song HK: Variation in use of blood transfusion in coronary artery bypass graft surgery. JAMA. 2010, 304: 1568-1575. 10.1001/jama.2010.1406.

2. MA Blajchman: Allogenic blood transfusions, immunomodulation and postoperative bacterial infection: do we have the answers yet. Transfusion. 37 (1997): 121-125

3. Alghamdi AA, Davis A, Brister S, Corey P, Logan A. Development and validation of Transfusion Risk Understanding Scoring Tool (TRUST) to stratify cardiac surgery patients according to their blood transfusion needs. Transfusion. 2006;46(7):1120-9.

4. Slight RD, Fung AKY, Alonzi C. Rationalizing blood transfusion in cardiac surgery: preliminary findings with a red cell volume-based model. Vox Sanguinis. 92(2):154-156.

5. Koch CG, Li L, Duncan AI, Mihaljevic T, Cosgrove DM, Loop FD. Morbidity and mortality risk associated with red blood cell and bloodcomponent transfusion in isolated coronary artery bypass grafting. Crit Care Med. 2006;34(6):160816.

6. Magedanz EH, Bodanese LC, Guaragna JC, Albuquerque LC, Martins V, Minossi SD. Risk score elaboration for mediastinitis after coronary artery bypass grafting. Rev Bras Cir Cardiovasc. 2010;25(2):154-9.

7. Friedman ND, Bull AL, Russo PL, Leder K, Reid C, Billah B. An alternative scoring system to predict risk for surgical site infection complicating coronary artery bypass graft surgery. Infect Control Hosp Epidemiol. 2007;28(10):1162-8.

8. Van Straten AH, Bekker MW, Soliman Hamad MA, van Zundert AA, Martens EJ, Schönberger JP. Transfusion of red blood cells: the impact on shortterm and long-term survival after coronary artery bypass grafting, a ten-year follow-up. Interact Cardiovasc Thorac Surg. 2010;10(1):37-42.

9. Murphy GJ, Reeves BC, Rogers CA, Rizvi SI, Culliford L, Angelini GD. Increased mortality, postoperative morbidity, and costs after red blood cell transfusion in patients having cardiac surgery. Circulation. 2007;116(22):2544-52.

10. Ranucci M. Allogeneic blood transfusions and infections after cardiac surgery. Am Heart J. 2007;153(5):e21.

11. Yoda M, Nonoyama M, Shimakura T. Autologous blood donation before elective off-pump coronary artery bypass grafting. Surg Today. 2004;34(1):213. 
12. Perthel M, El-Ayoubi L, Bendisch A, Laas J, Gerigk M. Clinical advantages of using minibypass system in terms of blood product use, postoperative bleeding and air entrainment: an in vivo clinical perspective. Eur J Cardiothorac Surg. 2007;31(6):1070-5.
13. Sellevold OF, Berg TM, Rein KA, Levang OW, Iversen OJ, Bergh K. Heparin-coated circuit during cardiopulmonary bypass. A clinical study using closed circuit, centrifugal pump and reduced heparinization. Acta Anaesthesiol Scand. 1994;38(4):372-9. 